\title{
Naturally Occurring Variations in Starch Synthase Isoforms in Rice Endosperm
}

(Received September 15, 2002)

\author{
Takayuki Umemoto, ${ }^{1, *}$ Noriaki Aoki ${ }^{1}$ and Takeshi Ebitani ${ }^{2}$ \\ ${ }^{1}$ National Institute of Crop Science (2-1-18, Kannondai, Tsukuba, Ibaraki 305-8518, Japan) \\ ${ }^{2}$ Toyama Agricultural Research Center (1124-1, Yoshioka, Toyama, Toyama 939-8153, Japan)
}

\begin{abstract}
We genetically analyzed cooked rice quality differences between japonica and indica rice varieties. By using backcross inbred lines, substitution, and near isogenic lines, we confirmed that at least 3 chromosomal regions affect cooked rice quality as measured by a Mido Meter. Each of these regions contains a locus for the starch synthase isoform, granule-bound starch synthase I (GBSSI), starch synthase I, and starch synthase IIa (SSIIa). We found naturally occurring variations in GBSSI (Wx protein) amount and SSIIa activity that greatly affect cooked rice quality by altering amylose content for GBSSI or amylopectin structure for SSIIa. In sensory tests, effects of GBSSI variation were clear even just after cooking, but those of SSIIa became clear only after cooling.

Key words: amylopectin, amylose, cooked rice quality, starch synthase, rice
\end{abstract}

The quality of cooked rice differs among rice varieties, and is one of the most important focuses in rice breeding in countries that cultivate rice, among which Japan is no exception. Differences in variety suggest genetic differences; however the genetics of cooked rice quality related to starch properties and metabolism have not been extensively studied. We genetically analyzed the relationship between the quality of cooked rice and starch properties.

We usually use sensory tests in our breeding programs to evaluate cooked rice quality. In such tests, about 10 varieties or new lines are cooked at once, then 20 to 30 testers eat and score each sample, comparing it to a standard variety. Such tests take time and work, which has led to the use of instrument tests based on the Mido Meter; in this case $30 \mathrm{~g}$ of polished rice is packed in a cartridge, soaked into hot water for $12 \mathrm{~min}$, and then surface shininess of cooked is measured. The Mido Meter manufacturer regards the exact mechanism behind the meter's operation as proprietary, preventing us from learning more about it, but we have noted a high correlation between Mido and sensory scores (Fig. 1). We used the Mido Meter to measure the quality of rice lines derived from a cross between japonica and indica subspecies, and used Mido scores for quantitative trait loci (QTL) analysis, a type of genetic analysis.

\section{QTL analysis of rice quality.}

QTL refers to loci controlling a quantitative trait. A quantitative trait is controlled by several genes rather than a single gene. QTL analysis locates genes regulating the trait of interest on chromosomes. To develop a gene mapping population for QTL analysis, 2 varieties whose traits of interest greatly differ are usually crossed. We used japonica variety Koshihikari, a favorite among Japanese people, and indica variety Kasalath, not a favorite among them. Koshihikari was backcrossed once and backcrossed

* Corresponding author (Tel.+81-298-38-8952, Fax. +81-29838-8837, E-mail: ume@affrc.go.jp). inbred lines consisting of 182 lines were developed as a gene mapping population ${ }^{1)}$ (Takeuchi et al., unpublished). Of this population, 180 lines of advanced $\mathrm{BC}_{1} \mathrm{~F}_{7}$ generation were used for QTL analysis of the Mido score.

The rice genome has 12 pairs of chromosomes. QTLs raising the Mido score with the Koshihikari genotype were detected on chromosomes 3 and 6. Two QTLs that raise the score with the Kasalath genotype were detected on chromosome 7. We focused on the QTL on chromosome 6, since the QTL region includes the loci for 3 starch synthase genes, -granule-bound starch synthase I (GBSSI or Wx), starch synthase I (SSI), and starch synthase IIa (SSIIa), surmising that variations or possible variations of these genes may be related to the Mido score.

\section{Confirmation of QTLs related to rice quality.}

Mido QTLs on chromosome 6 were confirmed by using substitution or near isogenic lines having a chromosomal segment of Kasalath with a Koshihikari genetic background (Fig. 2, left half). We also used near isogenic lines with the genetic background of another japonica variety, Nipponbare (Fig. 2, right half).

For the Koshihikari genetic background, substitution of the region including SSIIa locus decreased the Mido score. Additional substitution of the SSI region also adversely affected the score. The Mido score was notably decreased by further substitution of the GBSSI region. QTLs decreasing the Mido score for the Kasalath genotype by substituting each of the starch synthase loci were also seen for a Nipponbare genetic background. These confirm at least 3 genes controlling the Mido score exist on the short arm of chromosome 6. To determine whether these QTLs are based on naturally occurring variations of the 3 starch synthases and whether QTLs affect cooking quality in sensory tests, we conducted further studies mainly using a near isogenic line (NIL) and a substitution line (SL) for GBSSI and SSIIa with Nipponbare genetic background. 


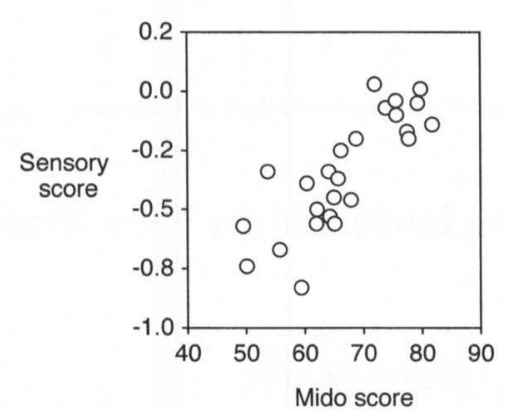

Fig. 1. Correlation between Mido score and sensory score of rice varieties.

All plant materials were cultivated at the Toyama Agricultural Research Center in 1994. Toyo Mido Meter MA-90B (Toyo Seimaiki Seisakusho) was used to measure the Mido score.

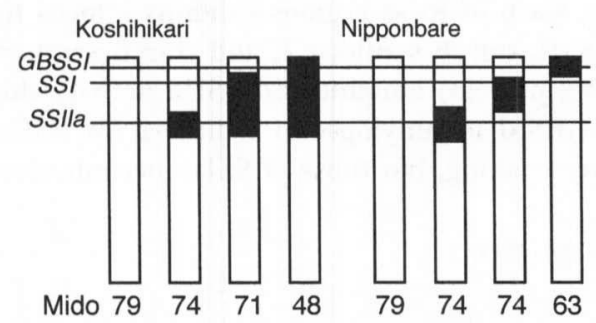

Fig. 2. Graphic genotype of chromosome 6 for near isogenic and substitution lines.

At left is Koshihikari and its NIL or SL having a chromosomal segment derived from Kasalath shown by the black rectangle. At right is Nipponbare and its NIL also having a part of Kasalath's chromosome. Mido scores are shown at the bottom.

\section{Starch synthase variations and rice quality.}

\section{1) Granule-bound starch synthase I (GBSSI).}

In amylose content, the 2 japonica varieties, Koshihikari and Nipponbare, clearly have less amylose than the indica Kasalath (Table 1). The near isogenic line having the $W x$ allele from Kasalath also has higher amylose content than the japonica. Since the ratio of amylose to amylopectin greatly affects starch properties, this difference in amylose content is expected to affect cooking quality. This difference is explained by the amount of GBSSI $(W x)$ protein, the enzyme synthesizes amylose.

The Kasalath $W x$ allele produced several times more $W x$ protein than the Koshihikari or Nipponbare $W x$ allele (Fig. 3A). Sano ${ }^{2)}$ first reported this major difference between japonica and indica, terming the $W x$ allele producing more $W x$ protein $W x^{a}$ and that producing less $W x$ protein $W x^{b}$. The cause of the difference between $W x^{a}$ and $W x^{b}$ function-a naturally occurring single base change at a splicing donor site in the first intron in $W x^{b}$-was found. ${ }^{3)}$ This leads to decreased production of mature mRNA reflected in decreased production of the $W x$ protein. Since DNA markers distinguishing $W x^{a}$ and $W x^{b}$ based on this

Table 1. Apparent amylose content of rice endosperm having $W x^{a}$ and $W x^{b}$ alleles.

\begin{tabular}{lcc}
\hline Variety & $W x$ genotype & Amylose content \\
\hline Koshihikari & $W x^{b}$ & $17.2 \pm 0.3$ \\
Nipponbare & $W x^{b}$ & $19.8 \pm 0.3$ \\
$W x^{a}$-NIL & $W x^{a}$ & $28.1 \pm 0.2$ \\
Kasalath & $W x^{a}$ & $29.5 \pm 0.2$ \\
\hline
\end{tabular}

Measured by the Juliano method. Values are the mean and standard deviation of 3 individual measurements from bulked samples. single base change are available, ${ }^{4,5}$ it is easy to detect which $W x$ allele a variety has (Fig. 3B). Does this difference in amylose content actually affect cooking quality? We compared Nipponbare and $W x^{a}$-NIL, lines having the $W x^{a}$ allele from Kasalath with a Nipponbare genetic background in sensory tests of cooked rice.

These tests compared $W x^{a}$-NIL to Nipponbare in appearance, hardness, stickiness, and overall preference. To Japanese testers, cooked $W x^{a}$-NIL rice had a poorer appearance, harder texture, and less stickiness, giving it a rather poor overall preference rating (Fig. 4).

\section{2) Starch synthase IIa (SSIIa).}

The SSIIa locus on chromosome 6, also called the alk locus, controls the alkali disintegration of rice grains. ${ }^{6} \mathrm{Ja}$ -
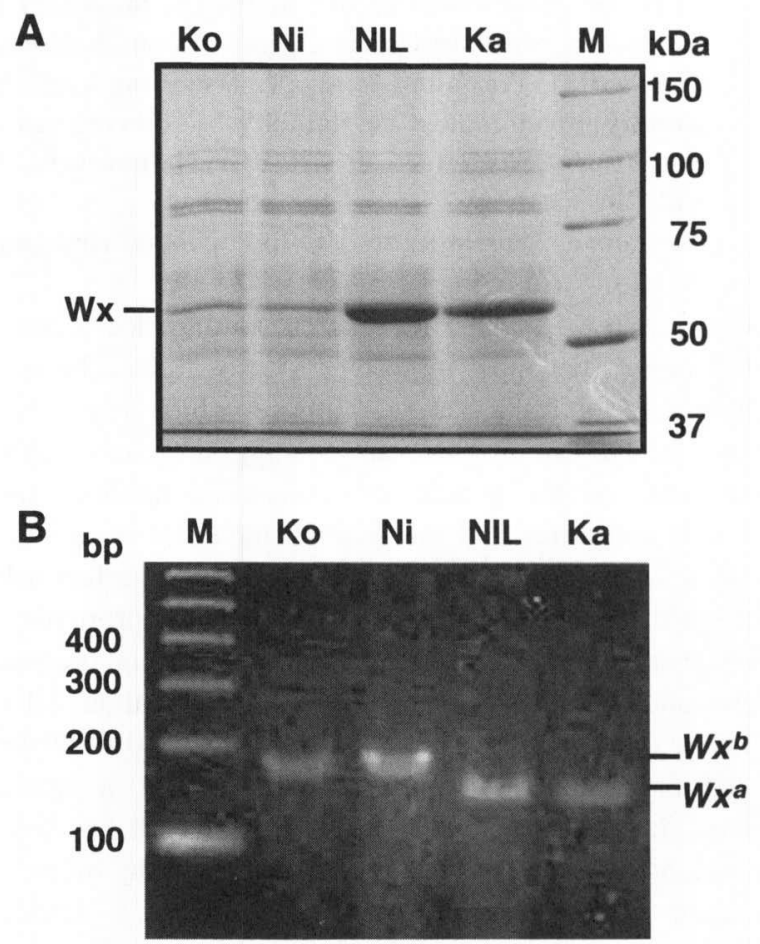

Fig. 3. (A) SDS-PAGE analysis of granule-bound proteins, and (B) dCAPS marker analysis of the $W x$ genotype $^{5}$ by agarose gel electrophoresis.

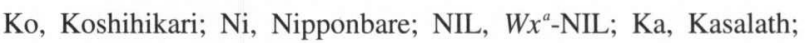
$\mathrm{M}$, molecular size marker.
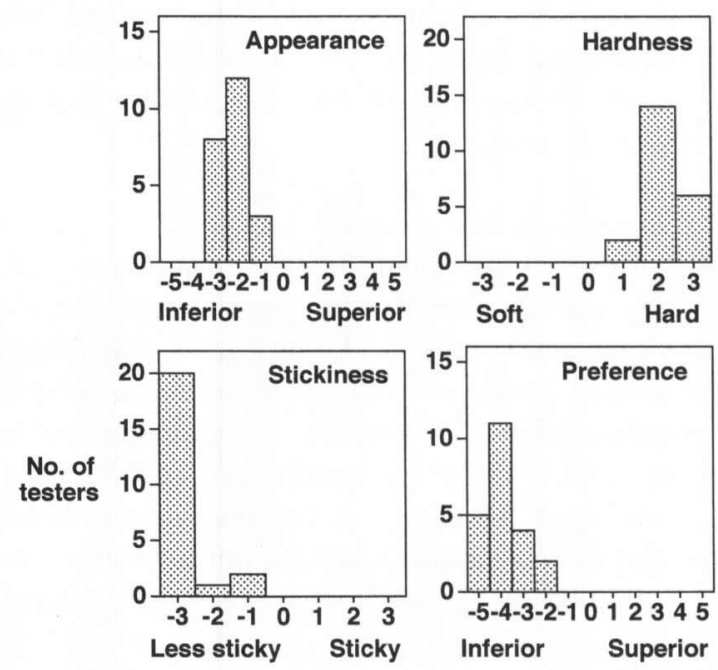

Fig. 4. Sensory evaluation of cooked $W x^{a}$-NIL rice compared to Nipponbare. 
ponica varieties tend to disintegrate in an alkaline solution of potassium hydroxide; however many indica varieties do not. ${ }^{7}$ We have shown genetically that alkali disintegration is most probably due to differences in amylopectin structure. ${ }^{8)}$ Compared to Kasalath, Nipponbare has a relatively greater number of short amylopectin chains-a difference also controlled at the same locus as alk. Since the SSIIa gene was also mapped at the same position, we suspect that SSIIa is identical to the alk gene.

We used a substitution line, SL-23, that has a Kasalath genotype about two-thirds of chromosome 6, including the SSIIa locus in the Nipponbare genetic background. As expected, SL-23 grains are, as with Kasalath, resistant to an alkaline solution (Fig. 5). We compared chain length distributions of amylopectin between them. Compared to Kasalath, Nipponbare had more short chains, and fewer edium-length chains (Fig. 6). In SL-23, the chain distribution resembles that of Kasalath. Very similar differences in chain length distributions of amylopectin were also reported with wheat that lacked SGP-I (SSIIa) protein ${ }^{9)}$ and antisense SSII lines of potato. ${ }^{10)}$

If the alk and SSIIa genes are the same, some difference should be seen in the function of SSIIa of Nipponbare and that of Kasalath because their alkali disintegration and amylopectin structure differ. We conducted native-PAGE active staining of starch synthase to identify differences in SSIIa function. We found an activity missing in Nipponbare that was detected in SL-23 (Fig. 7). Based on immunoblot analysis using an antibody raised against a synthesised SSIIa peptide, we confirmed that the activity band on the native gel was due to SSIIa (data not shown). Since Nipponbare and SL-23 have a functional difference in SSIIa, we regarded SSIIa as an alk gene candidate.

In sensory tests comparing Nipponbare and SSIIa-NIL, which still has a Kasalath chromosomal segment on chromosomes 5 and 8 (Fig. 8), the appearance score for SSIIa-NIL was only 0.5 point poorer than for Nipponbare, and hardness and stickiness did not differ between the two. Overall preference for SSIIa-NIL was slightly poorer than for Nipponbare. What happens if sensory tests are performed after cooked rice is cooled? We kept cooked

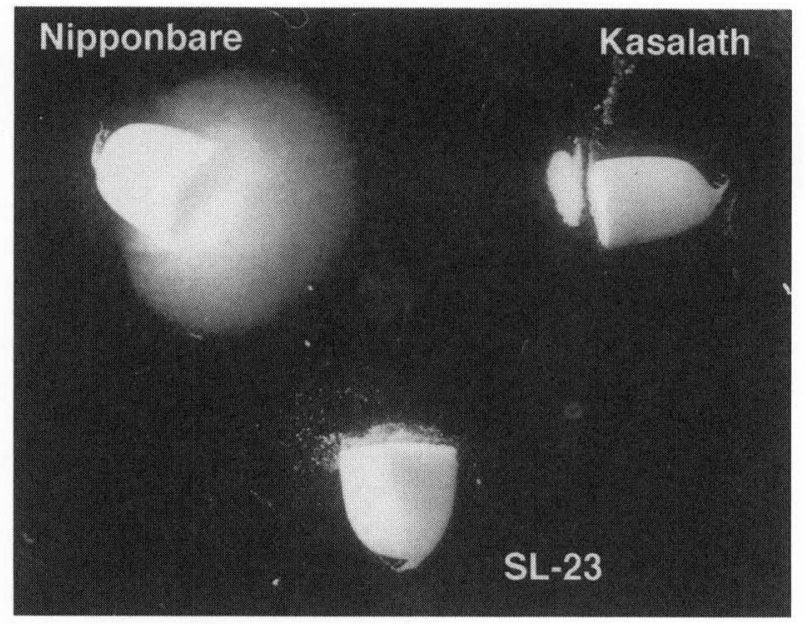

Fig. 5. Alkali disintegration of rice grains.

Halved rice grains were soaked in an alkaline solution of $1.3 \%$ potassium hydroxide $20 \mathrm{~h}$ at room temperature. samples overnight at $8^{\circ} \mathrm{C}$ and conducted sensory tests after samples reached room temperature. Compared to cooled Nipponbare, cooled SSIIa-NIL was judged to have a very poor appearance and a harder, less sticky texture, rating a very poor overall preference among Japanese testers.

Differences in alkali disintegration are thought to affect cooking quality, ${ }^{11)}$ but no near isogenic line for alk has been developed. Our results suggest that the difference in the SSIIa gene greatly affects cooking quality, probably

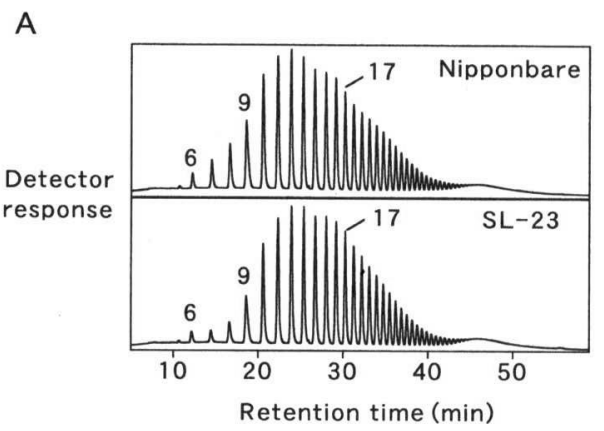

B

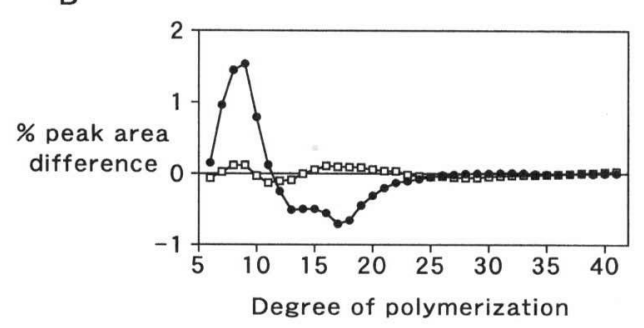

Fig. 6. Chain length distribution of amylopectin for Nipponbare and SL-23.

A) Amylopectin chain lengths were analyzed by HPAEC-PAD, B) Differences in chain length distribution were compared to those of Kasalath as the standard, represented on the X-axis. Nipponbare; $\square$, SL-23.

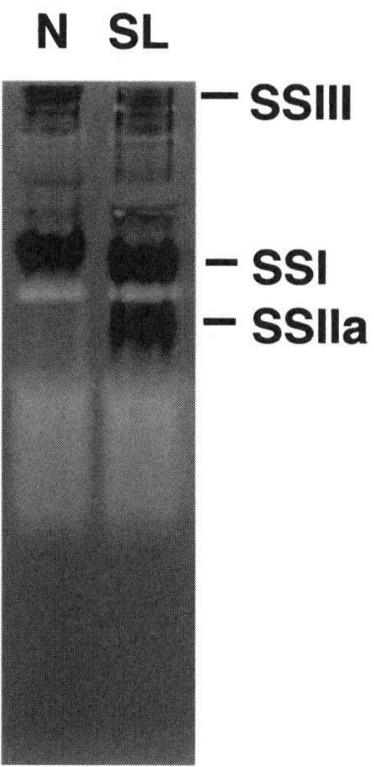

Fig. 7. Native-PAGE active staining for starch synthase in developing rice endosperm.

Soluble forms of starch synthase activity were separated by a gel containing $0.8 \%$ rabbit liver glycogen in 5\% acrylamide. The gel was incuvated in a buffer containing $1 \mathrm{mM} \mathrm{ADPG}$ for $24 \mathrm{~h}$ at $25^{\circ} \mathrm{C}$, then stained with acidified iodine solution. N, Nipponbare; SL, SL23. 

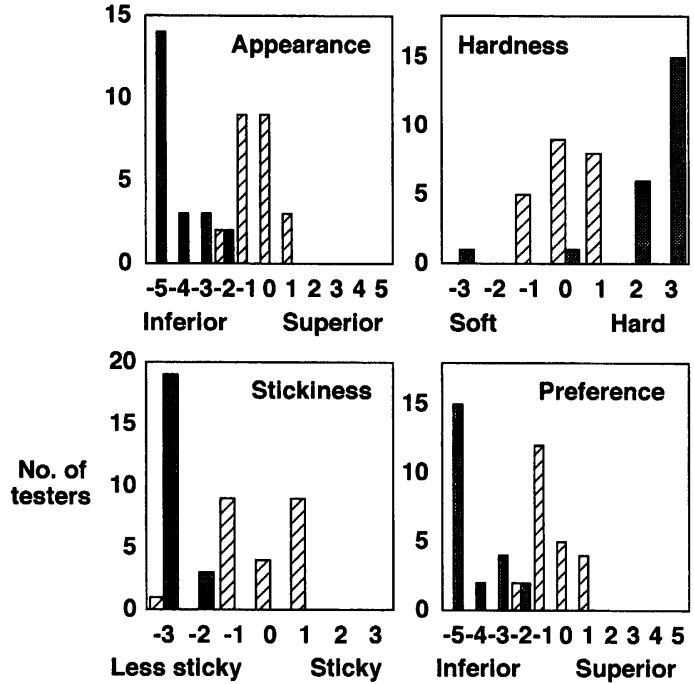

Fig. 8. Sensory evaluation of cooked SSIIa-NIL rice compared to Nipponbare.

Sensory tests were conducted just after rice has been cooked (hatched bars), and after samples had been kept over night at $8^{\circ} \mathrm{C}$ (shaded bars).

through altering the structure and physico-chemical properties of amylopectin, but only after cooked rice is cooled. These results should be confirmed after an advanced near isogenic line is developed for SSIIa.

We thank Profs. Yasunori Nakamura and Hikaru Satoh for their cooperation in SSIIa work, Dr. Masahiro Yano for providing rice lines, and staff members in the National Institute of Crop Science for aid with sensory tests of cooked rice.

\section{REFERENCES}

1) T. Yamamoto, F. Taguchi-Shiobara, Y. Ukai, T. Sasaki and M.
Yano: Mapping quantitative trait loci for days-to-heading, and culm, panicle and internode length in a $\mathrm{BC}_{1} \mathrm{~F}_{3}$ population using an elite rice variety, Koshihikari, as the recurrent parent. Breed. Sci., 51, 63-71 (2001).

2 ) Y. Sano: Differential regulation of waxy gene expression in rice endosperm. Theor. Appl. Genet., 68, 467-473 (1984).

3 ) Z.Y. Wang, F.Q. Zheng, G.Z. Shen, J.P. Gao, P. Snustad, M. G. Li, J.L. Zhang and M.M. Hong: The amylose content in rice endosperm is related to the post-transcriptional regulation of the waxy gene. Plant J., 7, 613-622 (1995).

4 ) N.M. Ayres, A.M. McClung, P.D. Larkin, H.F.J. Bligh, C.A. Jones and W.D. Park: Microsatellites and a single-nucleotide polymorphism differentiate apparent amylose classes in an extended pedigree of US rice germ plasm. Theor. Appl. Genet., 94, 773-781 (1997).

5 ) S. Yamanaka, Y. Fukuta, R. Ishikawa, I. Nakamura, T. Sato and Y.I. Sato: Phylogenetic origin of waxy rice cultivars in Laos based on recent observations for "Glutinous Rice Zone" and dCAPS marker of waxy gene. TROPICS, 11, 109-120 (2002).

6 ) M. Kudo: Genetical and thremmatological studies of characters, physiological or ecological, in the hybrids between ecological rice groups. Bull. Nat. Inst. Agric. Sci., Ser. D, 19, 1-84 (1968).

7 ) H. Morishima and H.I. Oka: Phylogenetic differentiation of cultivated rice, XXII. Numerical evaluation of the indicajaponica differentiation. Jpn. J. Breed., 31, 402-413 (1981).

8 ) T. Umemoto, M. Yano, H. Satoh, A. Shomura and Y. Nakamura: Mapping of a gene responsible for the difference in amylopectin structure between japonica-type and indica-type rice varieties. Theor. Appl. Genet., 104, 1-8 (1984).

9 ) M. Yamamori, S. Fujita, K. Hayakawa, J. Matsuki and T. Yasui: Genetic elimination of a starch granule protein, SGP-1, of wheat generates an altered starch with apparent high amylose. Theor. Appl. Genet., 101, 21-29 (2000).

10) A. Edwards, D.C. Fulton, C.M. Hylton, S.A. Jobling, M. Gidley, U. Rossner, C. Martin and A.M. Smith: A combined reduction in activity of starch synthase II and III of potato has novel effects on the starch of tubers. Plant J., 17, 251-261 (1999).

11) R.R. Little, G.B. Hilder and E.H. Dawson: Differential effect of dilute alkali on 25 varieties of milled white rice. Cereal Chem., 35, 111-126 (1958). 